\section{The effect of violating phrase structure rules and selectional restrictions on TEP patterns}

\author{
RICHARD M. WEIST and JEANETTE DOLEZAL* \\ University of Nebraska-Lincoln, Lincoln, Nebr. 68508
}

Two experiments were conducted to investigate the effects of violating phrase-structure rules and selectional restrictions on the TEP patterns in sentence learning tasks. It was found that violating selectional restrictions affected the between- and within-phrase pattern of responding. When phrase-structure rules were violated, the strings were found to be learned word by word. These findings were related to the problem of surface structure and deep structure analysis of sentence processing.

A number of investigators have shown that the violation of selectional restrictions generates semantically anomalous strings which are more difficult to perceive and to remember than normal sentences (e.g., Forster \& Ryder, 1971; Marks \& Miller, 1964). Violations of phrase structure rules result in totally ungrammatical strings which are more difficult to perceive and remember than anomalous strings (e.g., Downey \& Hakes, 1968; Miller \& Isard, 1963). Johnson (1965) found that the constituent structure of a sentence and embedding affected the pattern of transitional error probabilities (TEP). Between-phrase transitions had higher TEPs than within-phrase transitions. The transition from matrix to embedded sentence also resulted in high TEPs. The purpose of this research was to investigate the effects of violating phrase structure rules and selectional restrictions on the TEP pattern in two sentence learning tasks.

\section{EXPERIMENT 1}

Subjects

The Ss were 30 male and female introductory psychology students. Ten Ss were assigned to each of three experimental conditions. Experimental conditions were run in a random order, and Ss were assigned to conditions as they signed up to participate.

\section{MATERIALS}

The sentences used in this research had a SAAD matrix sentence and two embedded sentences. The matrix sentences were of the form: $\mathrm{NP}_{\text {subject }}$ + PAST $+\mathrm{V}_{\text {transitive }}+\mathrm{NP}_{\text {object }}$. The verbs were at least marked for animate $\langle\mathbf{A}\rangle$ and/or inanimate $\langle\mathrm{I}\rangle$ subjects and objects in the following combinations: (1) $<$ A or $I>$ subject and $\langle\mathrm{A}$ or $\mathrm{I}\rangle$ objects, e.g., signal; (2) $\langle A\rangle$ subject and $\langle A$ or I $\rangle$ object, e.g., eat; $(3)<A$ or I $>$ subject and $\langle\mathbf{A}\rangle$ object, e.g., strangle; (4) $\langle$ I $\rangle$ subject and $\langle$ I $\rangle$ object, e.g., overflow. One of the embedded

* Experiment 1 was conducted by the second author as an honors thesis. sentences resulted in a present participle modifying the subject NP. The verbs of these embedded sentences were also marked, e.g., gallop is marked for an $\langle A\rangle$ subject. The second sentence was embedded as a relative clause containing a predicate adjective which was semantically related to the subject NP of the matrix sentence. Nine sentences were generated, each containing nine words, e.g., The crawling snake that was slimy choked the rat; The sparkling champagne that was flat overflowed the glass.

Miller \& Isard's (1963) procedure was used to generate semantically anomalous strings in which subcategorization rules were maintained, but selectional restrictions were systematically broken, e.g., The galloping plane that was cuddly strangled the leaves; The grazing champagne that was healthy rustled the rider. In the third set of strings, called anagram strings by Miller and Isard, the original sentences were rearranged so as to violate phrase structure rules, e.g., The signaled the that plane airfield was lost soaring; Pleased was that the child kitten purring cuddly.

Johnson's (1965) paired-associate learning procedure was used. Word strings were paired with the digits 1-9 and presented on $3 \times 5$ in. cards at a $2: 2$-sec rate. Ten randomizations of the digit and digit-word string pairs were constructed. All Ss received $\mathbf{1 0}$ PA learning trials.

In general, there was very little responding on the first six trials, and only the last four trials were analyzed. This selection did not distort the

Table 1

TEPs for Sentences, Anomalous Strings, and Anagram Strings

\begin{tabular}{|c|c|c|c|c|c|c|c|c|}
\hline \multirow{2}{*}{$\begin{array}{l}\text { String } \\
\text { Type }\end{array}$} & \multicolumn{8}{|c|}{ Transitions } \\
\hline & 1 & 2 & 3 & 4 & 5 & 6 & 7 & 8 \\
\hline Sentence & 41 & 43 & 68 & 72 & 73 & 82 & 71 & 69 \\
\hline Anomalous & 66 & 71 & 82 & 88 & 96 & 96 & 94 & 88 \\
\hline Anagram & 83 & 91 & 97 & 98 & 98 & 99 & 99 & 99 \\
\hline
\end{tabular}

findings. TEPs were computed for each of the eight transitions in the nine word strings on Trials 7-10 and summed over trials. An analysis of variance was computed on these data, having the type of string (original sentence, semantically anomalous string, and anagram string) as a between-Ss variable, with 10 Ss nested under each type, and eight transitions within-Ss.

\section{Results}

The main effect of string type was significant, $F(2,27)=15.1, p<.001$. Anomalous strings were more difficult to learn than original sentences, $F(1,27)=12.9, p<.01$, and anagram strings were more difficult to learn than both original sentences and anomalous strings, $F(1,27)=17.1$, $\mathrm{p}<.001$. This finding supports previous research, e.g., Miller \& Isard (1963). The effect of transitions, $\mathrm{F}(7,189)=25.7, \mathrm{p}<.001$, and the interaction of Type by Transition, $\mathrm{F}(14,189)=2.4, \mathrm{p}<.01$, were significant. The TEPs for the eight transitions and three string types are shown in Table 1. In the original sentences, the shift from subject NP to relative clause and the deep break in the matrix sentence were responsible for relatively high TEPs. This observation was supported by the comparison of Transitions 1 and 2 with Transitions 3-8, which was significant, $F(1,189)=125.88$, $\mathrm{p}<.001$, and the comparison of Transition 6 with 7 and 8 , which was also significant, $F(1,189)=8.00$, $\mathrm{p}<.01$

When the strings were anomalous, the transition errors gradually increase from Transition 1 to 6 and then diminish slightly from Transition 6 to 8 . The comparison of Transition 6 vs 7 and 8 is not significant, $F(1,189)=$ $1.34, p>.10$. When phrase structure rules were violated, the function relating errors and transitions became relatively flat. For sentences, the TEPs were consistent within phrase and clause. The violation of selectional restrictions causes a gradual increase in TEP, such that between and within phrase and clause, transitions are less obvious.

In the paired-associate learning procedure, S must deal with a set of strings. This might have caused interference between strings. The fact that the TEP pattern for the anagram 
Table 2

Percentage of Words Reported Correctly in Nine Positions for Three String Types

\begin{tabular}{|c|c|c|c|c|c|c|c|c|c|}
\hline \multirow{2}{*}{$\begin{array}{l}\text { String } \\
\text { Type }\end{array}$} & \multicolumn{9}{|c|}{ Word Positions } \\
\hline & 1 & 2 & 3 & 4 & $\mathbf{5}$ & 6 & 7 & 8 & 9 \\
\hline Sentence & 99.8 & 96.3 & 99.6 & 95.8 & 93.5 & 83.8 & 57.7 & 58.6 & 53.8 \\
\hline Anomalous & 100.0 & 93.9 & 98.3 & 90.7 & 87.8 & 75.2 & 58.1 & 54.1 & 44.3 \\
\hline Anagram & 93.1 & 90.7 & 86.3 & 75.2 & 71.9 & 56.6 & 48.3 & 41.1 & 42.6 \\
\hline
\end{tabular}

strings was flat might have been due to the constant 10-trial procedure. A second experiment was designed to replicate these findings with string-by-string learning procedure.

\section{EXPERIMENT 2}

Subjects and Stimulus Material

The Ss were 15 students from the same population as those in Experiment 1. The set of nine sentences used in Experiment 1 was used, plus two additional sets of nine sentences of the same form. Anomalous and anagram strings were derived from the three sets of original sentences. Three lists were generated each list contained nine sentences, nine anomalous strings, and nine anagram strings. The anomalous and anagram strings were derived from different sets of original sentences; thus, no string within a list contained the same set of words. The strings within a list were randomly arranged for presentation, with the limitation that no string of the same type would be repeated.

\section{Procedure}

The strings were exposed to $\mathrm{S}$ by means of a tachistoscope. A white frame with an $X$ in the middle was exposed to $S$, and $S$ was instructed to focus on the $X$. Shortly after the $X$ appeared, it disappeared and a sentence was exposed for $1 \mathrm{sec}$ in the frame. The Ss were instructed to read the string aloud. Each string was exposed as many times as was necessary for $S$ to report the entire string. The words correctly reported in Positions 1-9 were recorded on each trial. The percentage of correct responses at each position was determined on the basis of the number of trials to criterion.

\section{Design}

The experiments consisted of three lists with five $\mathrm{Ss}$ nested under lists. Each $S$ responded to the three string types (sentences, anomalous, and anagram); nine strings were nested under each string type, and each string contained nine word positions. String type was made a within-S variable so that $S$ would not establish a set to respond to a particular string type.

\section{RESULTS AND DISCUSSION}

In general, the results were consistent with those of Experiment 1. The mean percentage of words correctly reported at each of the nine positions on the three string types are shown in Table 2. The effects of string type, word position, and the Type by Position interaction were significant, $p<.001$. The violation of selectional restrictions had a significant effect, $F(1,24)=8.90, p<.01$, but most of the variability was between string types, with phrase structure preserved vs phrase structure violated, $\mathbf{F}(1,24)=$ $117, \mathrm{p}<.001$. Violating selectional restrictions affected the pattern of responding over word positions, as seen by the interaction of Type by Position for the sentences vs anomalous strings, $F(8,192)=3.45$, $\mathrm{p}<.01$. The transition from NP to VP in the matrix sentence was less extreme when selectional restrictions were violated. When phrase structure rules are violated, the strings were learned word by word, and the interaction of Type by Position for the sentence and anomalous strings vs the anagram strings was significant, $F(8,192)=143, p<.001$.

These findings are related to the problem of surface structure and deep structure analysis of sentence processing. Johnson (1968) proposed that during sentence processing, words are coded by major category symbols ( $N, V, N P$, etc.). The final derived phrase marker (i.e., the surface structure) was used to represent the hierarchical arrangement of the code. This hierarchical schema represented the structure on which unitization or recoding was proposed to be based. assigned to words was never explained by Johnson. The present research shows that this analysis is inadequate. The words in both sentences and anomalous strings would be coded by the same set of major category symbols. Contrary to the expectations of this model, anomalous strings were more difficult to process than were sentences. Johnson (1968) was, of course, aware of research with anomalous strings and mentioned briefly a study of his own on the problem (pp. 433-434). Johnson considered the violation of selectional How major category symbols are restrictions as severely reducing word-to-word associations. If Johnson is claiming that selectional restriction can be equated with word-to-word association, then his position is inadequate on most linguistic analysis (e.g., Chomsky, 1965), and has also been shown to be inadequate in psychological investigations (e.g., Weisberg, 1971).

The present research is consistent with Fodor \& Garrett's (1967) lexical model on at least two points. In the first place, derivational complexity did not prove to be equivalent to psychological complexity. It was shown that adjectival embedding did not cause an increment in TEP, but the relative clause embedding did cause a TEP shift. Secondly, according to the lexical model, the process of recovering the deep structure begins with a lexical classification "... according to the base structure configurations ... [p. 295]." Selectional restrictions limit set of possible base structure configurations, and when these restrictions are violated, the job of sentence processing becomes more difficult. Violation of phrase structure rules reduces acquisition to a problem of serial list learning.

\section{REFERENCES}

CHOMSKY N Aspects of the theory of syntax. Cambridge, Mass: M.I.T. Press, 1965.

DOWNEY, R. G., \& HAKES, D. T. Some psychological effects of violating linguistic rules. Journal of Verbal Learning \& Verbal Behavior, 1968, 7,

$158-161$.
FODOR. J., \& GARRETT. M. Some syntactical determinants of sentential complexity. Perception \& Psychophysics, 1967, 2, 289-296.

FORSTER, K. I., \& RYDER, L. A. Perceiving the structure and meaning of sentences. Journal of Verbal Learning \& Verbal Behavior, 1971, 10, 285-296.

JOHNSON, N. The osychological reality of phrase structure rules. Journal of Verbal Learning \& Verbal Behavior, 1965, 4, 469-475.

JOHNSON, $\mathbf{N}$ Sequential verbal behavior. In T. R. Dix on and D. L. Horton (Eds.), Verbal behavior and general behavior theory. Englewood Cliffs, N.J: Prentice-Hall, 1968

MARKS, L. E., \& MILLER, G. A. The role of semantic and syntactic constraints in the memorization of English sentences. Journal of Verbal Learning \& Verbal Behavior, 1964, 3, 1-5.

MILLER, G. A., \& ISARD, S. Some perceptual consequences of linguistic rules. Journal of Verbal Learning \& Verbal Behavior, 1963, 2, 217-228.

WEISBERG, $R$. $W$. On sentence storage: The influence of syntactic versus semantic factors on intrasentence word association. Journal of Verbal Learning \& Verbal Behavior, 1971, 10, 631-644. 